\title{
ON ESSCHER TRANSFORMS IN DISCRETE FINANCE MODELS
}

BY

Hans Bühlmann, Freddy Delbaen, Paul Embrechts

Department of Mathematics, ETH Zürich

AND

Albert N. ShiryaeV

Steklov Mathematical Institute, Moscow

\section{General Technique}

The object of our study is

$$
S=\left(S_{0}, S_{1}, \ldots, S_{N}\right)=\left(S_{n}\right)_{0 \leq n \leq N}
$$

where each $S_{n}$ is a $m$-dimensional stochastic (real valued) vector, i.e.

$$
S_{n}=\left(S_{n}^{(1)}, S_{n}^{(2)}, \ldots, S_{n}^{(m)}\right)
$$

defined on a probability space $(\Omega, \mathcal{F}, P)$ and adapted to a filtration $\left(\mathcal{F}_{n}\right)_{0 \leq n \leq N}$ with $\mathcal{F}_{0}$ being the $\sigma$-algebra consisting of all null sets and their complements. In this paper we interpret $S_{n}^{(k)}$ as the value of some financial asset $k$ at time $n$.

Remark: If the asset generates dividends or coupon payments, think of $S_{n}^{(k)}$ as to include these payments (cum dividend process). Think of dividends as being reinvested immediately at the ex-dividend price.

\section{Definition 1}

(a) A sequence of random vectors

$$
\vartheta=\left(\vartheta_{0}, \vartheta_{1}, \ldots, \vartheta_{N}\right)=\left(\vartheta_{n}\right)_{0 \leq n \leq N}
$$

where

$$
\vartheta_{n}=\left(\vartheta_{n}^{(1)}, \vartheta_{n}^{(2)}, \ldots, \vartheta_{n}^{(m)}\right), \vartheta_{n} \text { is } \mathcal{F}_{n} \text {-measurable }
$$


is called a trading strategy. Since our time horizon ends at time $N$ we must always have $\vartheta_{N} \equiv 0$.

The interpretation is obvious: $\vartheta_{n}^{(k)}$ stands for the number of shares of asset $k$ you hold in the time interval $[n, n+1)$. You must choose $\vartheta_{n}$ at time $n$.

(b) The sequence of random variables

$$
\delta^{\vartheta}=\left(\delta_{n}^{\vartheta}\right)_{0 \leq n \leq N}
$$

where $\delta_{n}^{\vartheta}:=\left(\vartheta_{n-1}-\vartheta_{n}\right) S_{n}$ stands for the payment stream generated by $\vartheta$ $\left(\right.$ set $\left.\vartheta_{-1} \equiv 0\right)$.

\section{Remarks:}

i) Observe that $\vartheta_{n}, S_{n}$ are stochastic vectors, $\delta_{n}$ is a simple random variable and $\left(\vartheta_{n-1}-\vartheta_{n}\right) S_{n}$ has to be read as a scalar product. The best way to think of the above is to consider $S_{n}$ as the unit portfolio of all assets (you hold one unit of each cum dividend asset), $\vartheta_{n}$ your trading strategy for the period $[n, n+l)$ and $\vartheta_{n} S_{n}$ the value (at time $n$ ) of the portfolio held.

ii) Many papers in finance study the stream of discounted gains

$$
\vartheta_{n-1}\left(D_{n-1} S_{n}-S_{n-1}\right), \quad n=1,2, \ldots, N,
$$

for some discount factors $D_{0}, D_{1}, \ldots, D_{N-1}$ (where $D_{n-1}(>0)$ is adapted to $\mathcal{F}_{n-1}$ ) which may be sometimes more convenient. In particular each strategy then can be understood as an Itô-integral.

Our definition of payment stream needs no external definition of discount rates and is more natural from a cashflow point of view. Indeed, just think of an investor who at one time point takes a (long or short) position in the assets of his choice among $S^{(k)}(k=1,2, \ldots, m)$. He may change positions at every time point and has the obligation to liquidate all positions at time $N$ (hence $\vartheta_{N} \equiv 0$ ). Typically one of the assets is a bank account earning predictable interest. The latter means that the interest rate for the period $[k-1, k)$ is $\mathcal{F}_{k-1}$-measurable, for all $k \geq 1$.

\section{Definition 2}

1. Denote

(a) by $M:=\left\{\delta^{\vartheta} ; \vartheta\right.$ a trading strategy $\}$ the set of all payment streams generated by trading strategies,

(b) by $K:=\{X ; X \geq 0\}$ the set of all non-negative stochastic payment stream,

(c) by 0 the null payment stream and all equivalent payment streams.

2. We say that the trading strategy $\vartheta$ provides an arbitrage opportunity if $\delta^{\vartheta}=\left(\delta_{0}^{\vartheta}, \delta_{1}^{\vartheta}, \ldots, \delta_{N}^{\vartheta}\right) \geq 0, \quad$ and $\neq 0$ with positive probability. 
3. The absence of arbitrage opportunities then means

$$
M \cap K=\{0\} .
$$

We call (6) the No Arbitrage Condition (NAC).

\section{Remarks:}

i) Observe that we have defined no arbitrage based on the definition

$$
\delta_{n}^{\vartheta}:=\left(\vartheta_{n-1}-\vartheta_{n}\right) S_{n},
$$

i.e. $\delta_{n}^{\vartheta}$ is the $n$-th component of the payment stream.

ii) If for some predictable discounts $D_{n-1}(n=1,2, \ldots, N)$ we use the alternative definition

$$
\delta_{n}^{9}:=\vartheta_{n-1}\left(D_{n-1} S_{n}-S_{n-1}\right),
$$

i.e. $\delta_{n}^{y}$ is the stream of discounted gains, the condition (6) is called the Alternative No Arbitrage Condition (ANAC).

iii) The equivalence of the two no arbitrage conditions is discussed in the Appendix, from which one also can see the equivalence to the traditional definition of self-financing strategy with positive terminal value.

The basic idea of the whole pricing philosophy in finance consists in the construction of a linear functional $Q$ which strictly "separates" the payment streams obtained from trading strategies. To be more precise we want to prove the following theorem, which for the moment is stated loosely. Indeed, the particular spaces where $\left(S_{n}\right)_{0 \leq n \leq N}$ and $\left(\vartheta_{n}\right)_{0 \leq n \leq N}$ take values are not yet defined.

Theorem 1 The NAC (6) is a necessary and sufficient condition for the existence of a strictly positive linear functional $Q$ which is zero for all elements of $M$.

\section{Remarks:}

i) Because of its importance in finance the theorem above is usually referred to as the Fundamental Theorem of Asset Pricing.

ii) The necessity of (6) follows immediately. The difficult part to prove is: if (6) holds, then there exists a strictly positive linear functional $Q$ such that $Q\left(\delta^{\vartheta}\right)=0$ for all $\delta^{\vartheta} \in M$.

iii) Observe that under the NAC (6) one can prove by convexity arguments alone the existence of a nontrivial, non-negative functional $H$ such that $H\left(\delta^{\vartheta}\right)=0$ for all $\delta^{\vartheta} \in M$. Unfortunately this is of no help. In order to prove the existence of a strictly positive linear functional $Q$ such that $Q\left(\delta^{y}\right)=0$ for all $\delta^{y} \in M$ we must introduce a topology in our linear space. 
iv) It should be noted that many texts in finance treat the Fundamental Theorem of Asset Pricing in a finite probability space. Most of the proofs used in this context are - unfortunately - not valid for infinite probability spaces.

\section{TeChnique in a Normed Linear Space}

Let $L^{p}\left(P, \mathbb{R}^{m}, \mathcal{F}\right)$ be the space of real valued random vectors taking values in $\mathbb{R}^{m}$, measurable with respect to $\mathcal{F}$ and with integrable $p$-norm with respect to $P$. For the sequel we assume the following conditions

(C1) $\vartheta_{n} \in L^{2}\left(P, \mathbb{R}^{m}, \mathcal{F}_{n}\right), 0 \leq n \leq N$,

(C2) $S_{n} \in L^{\infty}\left(P, \mathbb{R}^{m}, \mathcal{F}_{n}\right), 0 \leq n \leq N$.

\section{Remarks:}

(C1) can be accepted as a reasonable restriction of strategies,

(C.2) is discussed in Schachermayer [7] who shows that it can be assumed without loss of generality.

Under (C1) and (C2) the payment streams $\delta^{\vartheta}$ are elements of $L^{2}\left(P, \mathbb{R}^{N+1}, \mathcal{F}_{N}\right)$, briefly denoted by $L_{N+1}^{2}$. Observe that $L_{N+1}^{2}$ is the space of stochastic $(N+1)$-vectors with finite second moments for all components. We do not require that the $n$-th component be $\mathcal{F}_{n}$-measurable. If we identify vectors which are almost surely equal, then $L_{N+1}^{2}$ is a Hilbert space with scalar product

$$
(X, Y)=E\left[\sum_{k=0}^{N} X_{k} Y_{k}\right] .
$$

We now prove that under (C1) and (C2) Theorem 1 holds.

\section{Proof of necessity of NAC:}

Assume $Q$ exists. If we have $\delta^{\vartheta} \geq 0$ and different from zero with positive probability, then $Q\left(\delta^{\vartheta}\right)>0$ contradicting that $Q$ is zero on $M$. Hence (6) is necessary.

Proof of sufficiency of NAC, part 1:

We follow the reasoning developed by Schachermayer [7] who proved the following Lemma:

Lemma 1 (Schachermayer's Lemma) If $M \cap K=\{0\}$, then also $\overline{M-K} \cap K=\{0\}$ where the closure is taken by $L^{2}$ limits.

Remark: Schachermayer proved the lemma for the Alternative No Arbitrage Condition (based on a stream of discounted gains). His proof can however be 
adapted to the No Arbitrage Condition (based on a stream of cash payments) as used by us. The basic argument in the proof of the lemma is as follows:

If for $0 \leq n \leq N$

$$
\left(\vartheta_{n-1}^{(r)}-\vartheta_{n}^{(r)}\right) S_{n}-{ }^{(r)} K_{n} \stackrel{L^{2}}{\longrightarrow} Y_{n} \geq 0,
$$

where ${ }^{(r)} K_{n} \geq 0$ for all $n$ and $r$, then one can find a trading strategy (x) $\vartheta \in L_{N+1}^{2}$ such that

$$
Y_{n} \leq\left({ }^{(\infty)} \vartheta_{n-1}-{ }^{(\infty)} \vartheta_{n}\right) S_{n} \quad \text { for all } n, \quad 0 \leq n \leq N .
$$

But, if $Y=\left(Y_{0}, Y_{1}, \ldots, Y_{n}, \ldots, Y_{N}\right) \not \equiv 0,{ }^{(\infty)} \vartheta$ would allow arbitrage.

The convergence is in $L_{N+1}^{2}$ to start with, but by passing to an appropriate subsequence one can argue by almost sure convergence.

Proof of sufficiency of NAC, part 2:

Take any strictly positive linear functional $L$ and define for every $\varepsilon>0$

$$
K_{\varepsilon}=K \cap\left\{X \in L_{N+1}^{2} ; \quad L(X) \geq \varepsilon \quad \text { and } \quad\|X\| \leq \frac{1}{\varepsilon}\right\}
$$

which is weakly compact and does not contain 0 . We can hence separate strictly $\overline{M-K}$ and $K_{\varepsilon}$; i.e. we have for some well chosen continuous linear functional $Q_{\varepsilon}: L_{N+1}^{2} \rightarrow \mathbb{R}$,

$$
Q_{\varepsilon}(Y)<Q_{\varepsilon}\left(X_{\varepsilon}\right) \text { for all } Y \in \overline{M-K} \text { and all } X \in K_{\varepsilon} .
$$

As $\overline{M-K}$ is a cone, we must have

$$
Q_{\varepsilon}(Y) \leq 0 \quad \text { and hence } \quad Q_{\varepsilon}\left(\delta^{\vartheta}\right)-Q_{\varepsilon}(X) \leq 0 \quad \text { for all } X \in K, \quad \delta^{\vartheta} \in M .
$$

As this implies $Q_{\varepsilon}\left(\delta^{\vartheta}\right)=0$, it follows that

$$
\begin{array}{ll}
Q_{\varepsilon}(X) \geq 0 & \text { for all } X \in K, \\
Q_{\varepsilon}(X)>0 & \text { for all } X \in K_{\varepsilon} .
\end{array}
$$

Take now $a_{k}>0$ and $\varepsilon_{k} \downarrow 0$ such that $\sum_{k=1}^{\infty} a_{k}\left\|Q_{\varepsilon_{k}}\right\|<\infty$ and define

$$
Q(X):=\sum_{k=1}^{\infty} a_{k} Q_{\varepsilon_{k}}(X)
$$

We then have

$$
\begin{array}{ll}
Q\left(\delta^{\vartheta}\right)=0 & \text { for all } \delta^{\vartheta} \in M, \\
Q(X)>0 & \text { for all } \quad X(\neq 0) \in K .
\end{array}
$$

The last line holds since every such $X$ lies in some $K_{\varepsilon_{k}}$ for $k$ sufficiently large. 


\section{RiESZ REPRESENTATION IN $L_{N+1}^{2}$}

Because $L_{N+1}^{2}$ is a Hilbert space, any continuous linear functional $Q$ defined on the whole $L_{N+1}^{2}$ can be represented as a scalar product. We formulate this fact as:

Theorem 2 Let $Q$ be a strictly positive linear functional on $L_{N+1}^{2}$.

(a) There is a unique element $\varphi \in L_{N+1}^{2}$ such that for all $X \in L_{N+1}^{2}$ we have

$$
Q(X)=E\left[\sum_{k=0}^{N} \varphi_{k} X_{k}\right] .
$$

(b) For all $k=0,1,2, \ldots, N$ we have $\varphi_{k}>0$.

(c) i) If $Q$ is restricted to the subspace of componentwise adapted vectors $X \in L_{N+1}^{2}$, then there is an adapted version of $\varphi$ (i.e. $\varphi_{n}$ is $\mathcal{F}_{n}$-measurable for every $\left.0 \leq n \leq N\right)-$ call it $\varphi^{(s)}-$ such that

$$
Q(X)=E\left[\sum_{k=0}^{N} \varphi_{k}^{(s)} X_{k}\right]
$$

for all $X$, adapted vectors in $L_{N+1}^{2}$.

ii) This $\varphi^{(s)}$ is unique, once $Q$ is given.

Terminology: Following Duffie [3] we call $\varphi$ a Deflator and $\varphi^{(s)}$ a Standard Deflator.

Remark: The interested reader should also note the links between price deflator and the supermartingale potential representation as given by Rogers [6].

\section{Proof:}

(a) Follows immediately from the Riesz Representation Theorem since $L_{N+1}^{2}$ is a Hilbert space.

(b) Since $Q$ is a strictly positive linear functional.

(c) i) Define $\varphi_{k}^{(s)}=E\left[\varphi_{k} \mid \mathcal{F}_{k}\right]$. Then for $X_{k}$, which is $\mathcal{F}_{k}$-measurable, we have

$$
E\left[\varphi_{k} X_{k}\right]=E\left[E\left[\varphi_{k} X_{k} \mid \mathcal{F}_{k}\right]\right]=E\left[\varphi_{k}^{(s)} X_{k}\right] .
$$


ii) Suppose that we have two Standard Deflators $\varphi^{(s)}$ and $\tilde{\varphi}^{(s)}$. For any $k$ define $\Delta_{k}=\tilde{\varphi}_{k}^{(s)}-\varphi_{k}^{(s)}$ which is $\mathcal{F}_{k}$-measurable. For $0 \leq n \leq N$ we must have

$$
E\left[\tilde{\varphi}_{k}^{(s)} \Delta_{k}\right]=E\left[\varphi_{k}^{(s)} \Delta_{k}\right]
$$

hence $E\left[\Delta_{k}^{2}\right]=0$, whence $\tilde{\varphi}_{k}^{(s)}=\varphi_{k}^{(s)}$ with probability 1 .

Convention: As we shall in the following apply $Q$ to vectors $X$ with adapted components only, we work from now on with the Standard Version $\varphi^{(s)}$ of the Deflator (and drop the superscript $s$ for convenience).

\section{The Martingale Property}

Let $S, Q, \varphi$ be as in the previous section and consider the value process of asset $l$,

$$
S^{(l)}=\left(S_{0}^{(l)}, S_{1}^{(l)}, S_{2}^{(l)}, \ldots, S_{N}^{(l)}\right) .
$$

The following theorem establishes the equivalence of Theorem 1 (together with its Riesz representation as expressed by Theorem 2) with the so called "Martingale property".

Theorem 3 Given a strictly linear functional $Q$ satisfying Theorem 1 and its representing $\varphi=\left(\varphi_{1}, \varphi_{2}, \ldots, \varphi_{N}\right)$, then we have that (a) $\left(\varphi_{k} S_{k}^{(l)}\right)_{0 \leq k \leq N}$ is a martingale (with respect to the original probability measure as assumed in Section 2) for all $l=1,2, \ldots, m$.

(b) Conversely any strictly positive $\varphi$ for which (a) holds defines a $Q$ satisfying Theorem 1 .

\section{Proof:}

(a) Assume existence of $Q$ with deflator $\varphi$. Choose for fixed $k$

$$
\begin{array}{rlrl}
\vartheta_{k-1}^{(l)} & =I_{F_{k-1}} & & \text { for some } F_{k-1} \in \mathcal{F}_{k-1}, \quad l, k \text { fixed, } \\
\vartheta_{j}^{(g)}=0 & \text { for all other values of } j, g .
\end{array}
$$

(For any Borel set $A, I_{A}$ denotes the indicator function of $A$ ). This defines a trading strategy $\theta$ which buys exactly one unit of asset $l$ at time $k-1$ provided $F_{k-1}$ occurs and, provided the unit has been bought, sells the unit at time $k$. Hence

$$
\delta_{k-1}^{(\vartheta)}=-S_{k-1}^{(l)} I_{F_{k-1}}, \quad \delta_{k}^{(\vartheta)}=S_{k}^{(l)} I_{F_{k-1}}
$$


are the only components of $\delta^{\vartheta}$ which differ from zero. $Q\left(\delta^{\vartheta}\right)=0$ can be written as

$$
-\int_{F_{k-1}} \varphi_{k-1} S_{k-1}^{(l)} d P+\int_{F_{k-1}} \varphi_{k} S_{k}^{(l)} d P=0 .
$$

Because equality (11) holds for all $F_{k-1} \in \mathcal{F}_{k-1}$ and $\varphi_{k-1} S_{k-1}^{(l)}$ is $\mathcal{F}_{k-1}$ measurable, we must have

$$
\varphi_{k-1} S_{k-1}^{(l)}=E\left[\varphi_{k} S_{k}^{(l)} \mid \mathcal{F}_{k-1}\right], \quad 1 \leq k \leq N,
$$

which is the martingale property.

(b) For the converse look at

$$
Q(X)=E\left[\sum_{k=1}^{N} \varphi_{k} X_{k}\right]
$$

We have to check that $Q\left(\delta^{\vartheta}\right)=0$ for all $\delta^{\vartheta} \in M$. Rewrite

$$
Q\left(\delta^{\vartheta}\right)=E\left[\sum_{k=0}^{N} \varphi_{k}\left(\vartheta_{k-1}-\vartheta_{k}\right) S_{k}\right]=E\left[\sum_{j=1}^{N} \vartheta_{j-1}\left(\varphi_{j} S_{j}-\varphi_{j-1} S_{j-1}\right)\right] \text {. }
$$

The second equality follows from summation by parts. Since $\varphi_{j} S_{j}-\varphi_{j-1} S_{j-1}$ represent the increments of a $m$-dimensional martingale the assertion $Q\left(\delta^{\vartheta}\right)=0$ follows immediately.

\section{Remarks:}

i) Note that the deflator $\varphi$ is universal, it turns all our asset price processes into martingales.

ii) Observe that also the (standard) deflator $\varphi_{k}$ is $\mathcal{F}_{k}$-measurable (but not $\mathcal{F}_{k-1}$-measurable) which in many applications in finance is considered to be a handicap (as it cannot be observed at time $k-1$ ) and hence gives rise to a change of measure. We are not pursuing this line of thought here. It will turn out to be of advantage to work with the original probability measure.

iii) Observe also that the probability distribution of the deflator $\varphi$ together with the filtration $\left(\mathcal{F}_{n}\right)_{n<N}$ can be understood as a summary characterizing the financial model. The task of modelling financial markets can hence be understood as the choice of a filtration and of the proper deflator and its probability distribution. 


\section{The Basic Problem}

Suppose that you know the original probability law $P$ of the stream of random vectors $S=\left(S_{0}, S_{1}, \ldots, S_{N}\right)$ where $S_{k}=\left(S_{k}^{(1)}, S_{k}^{(2)}, \ldots, S_{k}^{(m)}\right)$. How do you find a deflator $\varphi=\left(\varphi_{0}, \varphi_{1}, \ldots, \varphi_{N}\right)$ such that $\left(\varphi_{k} S_{k}^{(l)}\right)_{0 \leq k \leq N}$ is a martingale for every asset $l$ ?

(a) This problem represents the standard situation, whenever one wants to model asset prices in a given market.

(b) The problem is mostly formulated in a different language using a changed measure (which absorbs the deflator). As already mentioned we do not follow this route here.

\section{The EsSCher TRANSFORM}

The Esscher Transform always allows us to find a deflator which achieves the martingale property for all assets $l=1,2, \ldots, m$. We work here more conveniently with the so called span-deflator

$$
Y=\left(Y_{1}, Y_{2}, \ldots, Y_{N}\right) \quad \text { where } Y_{k}:=\frac{\varphi_{k}}{\varphi_{k-1}}, \quad k=1,2, \ldots, N .
$$

We also use the span-discounts

$$
D_{k-1}:=E\left[Y_{k} \mid \mathcal{F}_{k-1}\right] \text {. }
$$

Observe that $D_{k-1}>0$ and $D_{k-1}$ is $\mathcal{F}_{k-1}$-measurable, hence represents the discount in $[k-1, k)$ known at time $k-1$.

We assume that $D_{k-1}, k=1,2, \ldots, N$, are externally given. This is for instance the case if at each time $k-1$ there is a possibility to invest in an asset (or in a portfolio) that pays exactly 1 at each time $k$. The random variable $D_{k-1}$ is then the price of this asset (portfolio) at time $k-1$.

Theorem 4 If for all $k$ and all $\left(\mathcal{F}_{k-1}\right.$-measurable $) \alpha_{k-1}=\left(\alpha_{k-1}^{(1)}, \alpha_{k-1}^{(2)}, \ldots, \alpha_{k-1}^{(m)}\right)$ we have that $\alpha_{k-1}\left(D_{k-1} S_{k}-S_{k-1}\right)$ either vanishes with probability 1 or has both signs with positive probability, then there exists a value of $\alpha_{k-1}$ such that

$$
Y_{k}^{\left(\alpha_{k-1}\right)}=D_{k-1} \frac{e^{\alpha_{k-1} S_{k}}}{E\left[e^{\alpha_{k-1} S_{k}} \mid \mathcal{F}_{k-1}\right]}
$$

achieves the martingale probability, namely

$$
E\left[Y_{k}^{\left(\alpha_{k-1}\right)} S_{k}^{(l)} \mid \mathcal{F}_{k-1}\right]=S_{k-1}^{(l)} \quad \text { for all } l=1,2, \ldots, m .
$$


You can think of (12) as changing the original measure $P$ into $P^{\left(\alpha_{k-1}\right)}$ as follows:

$$
d P^{\left(\alpha_{k-1}\right)}\left[S_{k} \leq x \mid \mathcal{F}_{k-1}\right]=\frac{e^{\alpha_{k-1} x}}{E\left[e^{\alpha_{k-1} S_{k}} \mid \mathcal{F}_{k-1}\right]} d P\left[S_{k} \leq x \mid \mathcal{F}_{k-1}\right]
$$

which leads for appropriate $\alpha_{k-1}$ to the martingale condition

$$
D_{k-1} E^{\left(\alpha_{k-1}\right)}\left[S_{k}^{(l)} \mid \mathcal{F}_{k-1}\right]=S_{k-1}^{(l)} \quad \text { for all } l=1,2, \ldots, m .
$$

The equivalence of (15) and (13) follows immediately from (12).

\section{Definition 3}

(a) $P^{\left(\alpha_{k-1}\right)}$ as given by (14) is called the Esscher Transform of $P$ with coefficient $\alpha_{k-1}$.

(b) In view of the equivalence of (13) and (15) we also say that the spandeflator $Y_{k}^{\left(\alpha_{k-1}\right)}$ given by (12) achieves the Esscher Transform with coefficient $\alpha_{k-1}$.

\section{Remarks:}

i) Observe that you can apply the Esscher Transform either to $S_{k}$ or the increment $S_{k}-S_{k-1}$. The resulting span-deflator $Y_{k}^{\left(\alpha_{k-1}\right)}$ turns out to be the same.

ii) The condition in Theorem 4 is exactly the Alternative No Arbitrage Condition (ANAC) based on the payment streams $\delta^{\alpha}$ with $\delta_{n}^{\alpha}=\alpha_{n-1}\left(D_{n-1} S_{n}-S_{n-1}\right), n=1,2, \ldots, N$, as explained in Section 1.

Proof of Theorem 4: Look at the target function

$$
T_{k-1}(\alpha)=\ln E\left[e^{\alpha\left(D_{k-1} S_{k}-S_{k-1}\right)} \mid \mathcal{F}_{k-1}\right], \quad \alpha \in \mathbb{R}^{m} .
$$

Assuming existence and finiteness of $T_{k-1}(\alpha)$ in the neighbourhood of some $\alpha$, we can see that the if-condition of the theorem guarantees that the minimum of $T_{k-1}$ is assumed at an interior point $\alpha^{*}$. As $T_{k-1}\left(\alpha^{*}\right)=\min$, we must have

$$
\frac{\partial}{\partial \alpha^{(l)}} T_{k-1}\left(\alpha^{*}\right)=\frac{E\left[e^{\alpha^{*}\left(D_{k-1} S_{k}-S_{k-1}\right)}\left(D_{k-1} S_{k}^{(l)}-S_{k-1}^{(l)}\right) \mid \mathcal{F}_{k-1}\right]}{E\left[e^{\alpha^{*}\left(D_{k-1} S_{k}-S_{k-1}\right)} \mid \mathcal{F}_{k-1}\right]}=0
$$

or

$$
E\left[Y_{k}^{\left(\alpha^{*} D_{k-1}\right)} S_{k}^{(l)} \mid \mathcal{F}_{k-1}\right]=S_{k-1}^{(l)} \quad \text { for } l=1,2, \ldots, m
$$


which is exactly the martingale condition (13). Observe that the argument is valid for all discounts which satisfy the condition in Theorem 4 . At this point it should also be clear that the $\log$ in defining $T_{k-1}(\alpha)$ is introduced to ensure that the derivative produces the Esscher Transform.

The mathematics still to be done is proving that $\alpha^{*}$ can be chosen to be $\mathcal{F}_{k-1}$-measurable. This can be done e.g. by the reasoning as found in Rogers [5]. Hence the Esscher Transform with coefficient $\alpha_{k-1}=\alpha^{*} D_{k-1}$ solves our basic problem defined in Section 5 .

Remark: The Esscher Transform can also be used for explicit calculation of prices. The reader who is interested in an explicit derivation of the BlackSchoies formula using the Esscher Transform, should consult Gerber and Shiu [4].

\section{Why ESSCHER TRANSFORM?}

From Section 6 it is evident that Esscher Transforms are convenient. Are there further reasons for choosing this transform? The following economical reasoning may be an additional argument.

In this section there is a change of notation; we interpret $\left(S_{0}, S_{1}, \ldots, S_{N}\right)$ as a sequence of random variables $S_{k}$. Until now the vector $S_{k}=\left(S_{k}^{(1)}, S_{k}^{(2)}, \ldots, S_{k}^{(m)}\right)$ was interpreted as values of the $m$ assets in the market. Now you should think of $S_{k}$ as the total aggregate market value of all assets, i.e. their unit values multiplied by their volumes

$$
S_{k}=\sum_{l=1}^{m} V_{k-1}^{(l)} S_{k}^{(l)}
$$

where $V_{k-1}^{(l)}$ denotes the volume of asset $l$ in $[k-1, k)$. The discounted increase in $[k-1, k)$

$$
W_{k}=D_{k-1} S_{k}-S_{k-1}=\sum_{l=1}^{m} V_{k-1}^{(l)}\left(D_{k-1} S_{k}^{(l)}-S_{k-1}^{(l)}\right)
$$

is the object of our study in this section. For simplicity we assume that for all $l, V_{k-1}^{(l)}$ are chosen at time $k-1$ and remain constant in $[k-1, k)$, hence in particular all $V_{k-1}^{(l)}$ are $\mathcal{F}_{k-1}-$ measurable.

We want to study the Pareto optimal allocation of $W_{k}$. This justifies our simplifying assumption that $S_{k}, S_{k-1}$ are only one dimensional random variables. It is well known that Pareto optimal allocations are always functions of the aggregate value (see e.g. Borch [1]). 
In order to distribute $W_{k}$ in a Pareto optimal way among $M$ investors, we characterize each investor $j$ by his utility function

$$
u_{j}(x)=\frac{1}{\gamma_{j}}\left(1-e^{-\gamma_{j} x}\right), \quad j=1,2, \ldots, M .
$$

We hence suppose that you may describe the preferences of each investor $j$ by an exponential utility function, where $\gamma_{j}$ is the risk aversion of investor $j$, or equivalently, $\frac{1}{\gamma_{j}}$ is the risk tolerance of investor $j$. The feasibility of this assumption is discussed at the end of this section.

A Pareto optimal allocation

$$
\left(W_{k}^{(1)}, W_{k}^{(2)}, \ldots, W_{k}^{(j)}, \ldots, W_{k}^{(M)}\right)
$$

can be obtained as a Price Equilibrium. Denote by Price $\left[W_{k}\right]$, the $\mathcal{F}_{k-1}$ measurable functional assigning a price to the random variable $W_{k}$ held during the time interval $[k-1, k]$. A Price Equilibrium is achieved if at time $k-1$ the following conditions are satisfied.

(a) For each $j$

$$
E\left[u_{j}\left(W_{k}^{(j)}-\operatorname{Price}\left[W_{k}^{(j)}\right]\right) \mid \mathcal{F}_{k-1}\right]
$$

achieves a maximum among all possible random variables $W_{k}^{(j)} \in L^{2}$.

(b) The allocation has to satisfy

$$
\sum_{j=1}^{M} W_{k}^{(j)}=W_{k} .
$$

If we explicitly define the price functional by the span-deflator $Y_{k}$, we have

$$
\operatorname{Price}\left[W_{k}^{(j)}\right]=E\left[Y_{k} W_{k}^{(j)} \mid \mathcal{F}_{k-1}\right]
$$

then Borch's condition (see Borch [1]) must be satisfied, i.e.

$$
u_{j}^{\prime}\left(W_{k}^{(j)}-E\left[Y_{k} W_{k}^{(j)} \mid \mathcal{F}_{k-1}\right]\right)=C_{j} Y_{k} \quad \text { for all } j=1,2, \ldots, M,
$$

where $C_{j}$ must be $\mathcal{F}_{k-1}$-measurable for all $j=1,2, \ldots, M$. Using the exponential form of the utility functions, $u_{j}^{\prime}(x)=e^{-\gamma_{j} x}$, (23) turns into

$$
e^{-\gamma_{j} W_{k}^{(j)}}=A_{j} Y_{k}, \quad j=1,2, \ldots, M,
$$


with $A_{j}$ positive and $\mathcal{F}_{k-1}$-measurable. Hence by taking logarithms

$$
-W_{k}^{(j)}=\frac{1}{\gamma_{j}} \ln A_{j}+\frac{1}{\gamma_{j}} \ln Y_{k} .
$$

Now sum over $j$ and use the abbreviation $\frac{1}{\gamma}:=\sum_{j=1}^{M} \frac{1}{\gamma}$, the sum of all risk tolerance units. Then you find ${ }^{\gamma}-W_{k}^{j=1}=B_{k}+\frac{1}{\gamma} \ln Y_{k}$ or $-\gamma W_{k}=\gamma B_{k}+\ln Y_{k}$, where $B_{k}$ is $\mathcal{F}_{k-1}$-measurable. The condition $E\left[Y_{k} \mid \mathcal{F}_{k-1}\right]=D_{k-1}$ allows you to get the value of $B_{k}$ and

$$
Y_{k}=D_{k-1} \frac{e^{-\gamma \boldsymbol{W}_{k}}}{E\left[\boldsymbol{e}^{\left.-\gamma \boldsymbol{W}_{k} \mid \mathcal{F}_{k-1}\right]}\right.} .
$$

\section{Discussion:}

i) We have found an economic reason to use the Esscher Transform. Indeed with our interpretation of $S_{k}$ in this section we get an economic interpretation for the Esscher parameter. We have $W_{k}=\sum_{l=1}^{m} V_{k-1}^{(l)}\left(D_{k-1} S_{k}^{(l)}-S_{k-1}^{(l)}\right)$, hence

$$
-\gamma W_{k}=-\sum_{l=1}^{m} \gamma V_{k-1}^{(l)}\left(D_{k-1} S_{k}^{(l)}-S_{k-1}^{(l)}\right) .
$$

ii) The economic argument has led us to the Esscher Transform with spandeflator

$$
Y_{k}^{\left(-\gamma D_{k-1} V_{k-1}\right)}
$$

with $V_{k-1}=\left(V_{k-1}^{(1)}, V_{k-1}^{(2)}, \ldots, V_{k-1}^{(l)}, \ldots, V_{k-1}^{(m)}\right)$. Hence we have found the Esscher parameter

$$
\alpha_{k-1}=-\gamma D_{k-1} V_{k-1} .
$$

On the other hand we have found in Section 6

$$
\alpha_{k-1}=D_{k-1} \alpha^{*}
$$

where $\alpha^{*}$ minimizes $T_{k-1}(\alpha)$ as defined by (16). Comparing the two results we must have

$$
\alpha^{*}=-\gamma V_{k-1} .
$$

Hence we can interpret the value $\alpha^{*}$ which minimizes $T_{k-1}(\alpha)$ as follows:

(a) $\alpha^{*}$ is proportional to the volume vector, and

(b) the (negative) proportionality factor for all components is $\gamma$ where $\frac{1}{\gamma}$ equals the sum of the risk tolerance units of all investors. 
iii) Obviously this conclusion is only correct for an economy where all investors have an exponential utility. Nevertheless you should note that the risk aversion $\gamma_{i}$ of each investor is allowed to change over time. So, if changes of values from one period to the next are not extremely large, one can think of the exponential utility functions as approximations to general (risk averse) utility functions. For an argument to understand this approximation we refer to Bühlmann [2]. It would be interesting to learn how the relation (25) compares with practical observations.

\section{Appendix: Equivalence of No Arbitrage Condition and Alternative No Arbitrage Condition}

We have two possibilities to define arbitrage opportunities.

First case: With the strategy $\vartheta=\left(\vartheta_{0}, \vartheta_{1}, \ldots, \vartheta_{N}\right)$ we define the payment stream

$$
\delta^{\vartheta}=\left(\delta_{n}^{\vartheta}\right)_{0 \leq n \leq N} \quad \text { with } \quad \delta_{n}^{\vartheta}:=\left(\vartheta_{n-1}-\vartheta_{n}\right) S_{n}
$$

An arbitrage opportunity is given by a strategy $\vartheta$ such that $\delta^{\vartheta}$ does not vanish and $\delta_{n}^{\vartheta} \geq 0$ for $n=1,2, \ldots, N$.

Alternative case: The same definition is applied to the stream of discounted gains

$$
\delta^{\vartheta}=\left(\delta_{n}^{\vartheta}\right)_{0 \leq n \leq N} \quad \text { with } \quad \delta_{n}^{\vartheta}:=\vartheta_{n-1}\left(D_{n-1} S_{n}-S_{n-1}\right)
$$

for some given discounts $D_{0}, D_{1}, \ldots, D_{N-1}$.

For both cases we refer to an arbitrage opportunity in $[0, N]$.

Definition 4 We say that there is an arbitrage opportunity in $[n-1, n]$ if there is a $\vartheta$ of the form $\left(0,0, \ldots, 0, \vartheta_{n-1}, 0, \ldots, 0\right)$ which allows arbitrage in $[0, N]$. More explicitly if there is a $\vartheta_{n-1}$ such that,

in the first case:

$$
\begin{aligned}
-\vartheta_{n-1} S_{n-1} & \geq 0, \\
\vartheta_{n-1} S_{n} & \geq 0,
\end{aligned}
$$

where at least one of the two left sides does not vanish;

in the alternative case:

$$
\vartheta_{n-1}\left(D_{n-1} S_{n}-S_{n-1}\right) \geq 0
$$

where the left side does not vanish.

Lemma 2 In both cases there is an arbitrage opportunity in $[0, N]$ if and only if there is an arbitrage opportunity in one of the intervals $[n-1, n], n=1,2, \ldots, N$. 
Proof: We only have to show that the existence of an arbitrage opportunity in $[0, N]$ implies existence of an arbitrage opportunity in one of the intervals $[n-1, n] ; n=1,2, \ldots, N$. In the alternative case the statement holds by definition, which leaves us with the first case. Assume arbitrage in $[0, N]$ in this case, hence there exists a $\vartheta$ such that

$$
\begin{aligned}
-\vartheta_{0} S_{0} & \geq 0, \\
\left(\vartheta_{0}-\vartheta_{1}\right) S_{1} & \geq 0, \\
\vdots & \\
\left(\vartheta_{N-2}-\vartheta_{N-1}\right) S_{N-1} & \geq 0, \\
\vartheta_{N-1} S_{N} & \geq 0,
\end{aligned}
$$

where not all the left sides may vanish.

We proceed by induction of the interval length $[0, N]$ :

(a) If $\vartheta_{N-1} S_{N-1}<0$ on $A$ with $P[A] \neq 0$ take $\vartheta_{N-1}=\vartheta_{N-1} I_{A}$ which achieves arbitrage in $[N-1, N]$.

(b) If $\vartheta_{N-1} S_{N-1} \equiv 0$ we have either arbitrage in $[N-1, N]$ if $\vartheta_{N-1} S_{N} \not \equiv 0$ or arbitrage in $[0, N-1]$ if $\vartheta_{N-1} S_{N} \equiv 0$.

(c) If $\vartheta_{N-1} S_{N-1} \geq 0$ (not vanishing), then also $\vartheta_{N-2} S_{N-1} \geq 0$ (not vanishing), hence we have arbitrage in $[0, N-1]$.

From (a), (b), (c) it follows that we have either arbitrage in $[N-1, N]$ or arbitrage in $[0, N-1]$ which proves recursion from $N$ to $N-1$.

Theorem 5 No Alternative Arbitrage implies No Arbitrage.

Proof: We show that any arbitrage opportunity allows to find an alternative opportunity. Because of Lemma 2 we can restrict ourselves to arbitrage opportunities in a unit interval $[n-1, n]$, e.g. $[0,1]$. An arbitrage opportunity means a strategy $\vartheta$ such that $-\vartheta_{0} S_{0} \geq 0, \vartheta_{0} S_{1} \geq 0$, with not both left sides vanishing. This implies for arbitrary discount $D_{0}>0$

$$
\vartheta_{0}\left(D_{0} S_{1}-S_{0}\right) \geq 0
$$

not vanishing, hence an alternative arbitrage opportunity.

Theorem 6 No Arbitrage implies No Alternative Arbitrage with conveniently chosen discounts under the conditions used for proving Theorem 1.

Proof: The main point of the proof is that we need the Fundamental Theorem of Asset Pricing. We argue again only in the interval $[0,1]$. The Fundamental Theorem of Asset Pricing guarantees the existence of the spandeflator $Y_{1}$ such that

$$
E\left[Y_{1} S_{1}^{(l)} \mid \mathcal{F}_{0}\right]=S_{0}^{(l)} \text { for all } l=1,2, \ldots, m .
$$


Hence $\vartheta_{0} E\left[Y_{1} S_{1} \mid \mathcal{F}_{0}\right]=\vartheta_{0} S_{0}$ for all $\vartheta_{0}$ which are $\mathcal{F}_{0}$-measurable

$$
E\left[Y_{1} \vartheta_{0} S_{1}-\vartheta_{0} S_{0} \mid F_{0}\right]=0
$$

which implies by the mean value theorem that either $\vartheta_{0}\left(D_{0} S_{1}-S_{0}\right) \equiv 0$ or $\vartheta_{0}\left(D_{0} S_{1}-S_{0}\right)$ has both signs with probability 1 for the $D_{0}=E\left[Y_{1} \mid \mathcal{F}_{0}\right]$. This is exactly the Alternative No Arbitrage Condition.

\section{ACKNOWLEDGEMENT}

The authors would like to thank the referees for their careful reading of a first version of this paper. Their comments led to numerous improvements.

\section{REFERENCES}

[1] BoRCH, K. (1962). Equilibrium in a reinsurance market. Econometrica 30, 424-444.

[2] BühlmanN, H. (1984). The general economic premium principle. ASTIN Bulletin 14, 13-21.

[3] Duffie, D. (1996). Dynamic Asset Pricing Theory, 2nd edition, Princeton University Press.

[4] Gerber, H.U. and Shiu, Elias S.W. (1994). Option pricing by Esscher transforms. Transactions of the Society of Actuaries, vol. XLVI, pp. 99-140.

[5] Rogers, L.C.G. (1994). Equivalent martingale measures and no-arbitrage. Stochastics and Stochastics Reports 51, 41-49.

[6] RogERS, L.C.G. (1997). The potential approach to the term structure of interest rates and their foreign exchange rates. Mathematical Finance 7, 157-176.

[7] SCHACHERMAYeR, W. (1992). A Hilbert space proof of the fundamental theorem of asset pricing in finite discrete time. Insurance: Mathematics and Economics 11, 249-257.

HANS BÜHLMANN,

Freddy Delbaen,

PAul EMBRechts

Department of Mathematics

ETH Zürich

CH - 8092 Zürich

Switzerland
Albert SHIRYAEV

Steklov Mathematical Institute

Ulitza Vavilova 42

Moscow 117966, GSP-1

Russia 